\title{
THE EVOLUTION OF CLINICAL RESEARCH AND THE OPEN REGISTRATION
}

The development of science and technology that has occurred through the decades finds in clinical research the great possibility of translating findings applicable to human health. Clinical trials have given everyone the opportunity to have access to health services. On the other hand, many pharmaceutical industries have stained clinical research with doubt and improbability. The results of controlled clinical trials and the selective, manipulated scientific publications with wrong conclusions led to inappropriate clinical practice, mainly favoring the economic aspect. In 2005, the International Committee of Medical Journal Editors (ICMJE) supported by World Association of Medical Editors started requiring that all clinical trials be registered in the database ClinicalTrials.gov as a prerequisite for publication. In 2006, the World Health Organization (WHO) created the International Clinical Trial Register Platform (ICTRP), gathering several register centers of the world, and demanded that all researchers and pharmaceutical industries register clinical trials. The registration of clinical trials implies an additional step of clinical research towards transparency, ethics and impartiality, resulting in real evidence which will direct changes in clinical practice and in the health situation.

Thus, The Journal of Venomous Animals and Toxins including Tropical Diseases follows the policies of both WHO and ICMJE for clinical trial registration and recognizes the importance of those initiatives for the international dissemination of clinical research information in open access system. Accordingly, only articles about trials previously registered in one of the Clinical Trial Registers that meet WHO and ICMJE requirements will be accepted for publication, starting in 2007. The list of registers accepted by $\mathrm{WHO}$ and ICMJE is available at ICMJE website (www.icmje.org). The Trial Registration Number should be published at the end of the Abstract. The current issue brings a special Review Article by Professor Caramori, who is in charge of the Clinical Research Unity at Botucatu Medical School, UNESP.

CORRESPONDENCE TO:

BENEDITO BARRAVIERA, Caixa Postal, 576, 18.618-000, Botucatu, SP, Brasil. Email: bbviera@jvat.org.br. 